Casos Clínicos

Arch. Esp. Urol. 2009; 62 (2): 147-150

\section{TUMOR CARCINOIDE PRIMARIO DE RIÑÓN. A PROPÓSITO DE UN CASO}

\author{
Rául Espinosa, Eduardo Bercowsky, José M. Benet', \\ Ramón Gibernau y Miguel Gascón. \\ Servicio de Urología y Servicio de Anatomía Patológical. \\ Hospital Mateu Orfila. Mahón. Menorca. España.
}

Resumen.- El tumor carcinoide primario de riñón es un tumor de presentación inusual, habiendo sido reportados 40 casos en la literatura. Describimos la presentación de una paciente con un tumor carcinoide primario de riñón.

OBJETIVO: Aportar a la literatura un nuevo caso de tumor carcinoide renal, por lo infrecuente del mismo y su forma de presentación ajena en ocasiones a síntomas urinarios, sino más bien asociado a trastornos de otros aparatos que enmascaran el cuadro clínico, donde es más frecuente dicho tumor.

MÉTODOS: Estudiar a una paciente de 80 años de edad, que es ingresada en el Servicio de Digestivo con un síndrome anémico, asociado a un cuadro clínico diarreico de una semana de evolución. Después de estudios humorales, heces fecales y de imagen se detecta masa sólida parenquimatosa renal izquierda; siendo enviada a nuestro

\section{CORRESPONDENCIA}

Rául Espinosa

Servicio de Urología

Hospital Mateu Orfila

Mahón. Menorca. (España).

raul.espinosa@hgmo.es

Trabajo recibido: 28 de agosto 2008 servicio donde se le realiza nefrectomía radical. Después de 8 meses de evolución postquirúrgica su trayectoria es satisfactoria.

RESULTADOS: El tumor carcinoide renal es una entidad infrecuente, que se engloba dentro de los tumores neuroendocrinos, descritos hace 100 años por Lubart, derivándose de las células de Kuchitsky, productoras de hormonas polipéptidos y aminas biógenas; siendo su frecuencia mayor en el aparato digestivo (62-67\%) y respiratorio (22-27\%). Normalmente estos tumores cuando se diagnostican a tiempo evolucionan favorablemente.

CONCLUSIONES: El tumor carcinoide renal primario es una entidad infrecuente dentro de los fumores endocrinos. Debemos señalar que este caso su forma de presentación fue como anemia, diarreas y sangre oculta en heces fecales en la analítica.

Palabras clave: Enfermedad renal. Carcinoma renal. Tumor carcinoide.

Summary.- The primary carcinoid tumor of the kidney is a tumor of unusual presentation, 40 cases have been reported in the literature. We describe the presentation of a patient with a primary carcinoid tumor of the kidney.

OBJETIVES: To report a new case of renal carcinoid tumor, a rare tumor sometimes presenting without uri-nary tract symptoms, but rather associated with disorders of other organs that mask the clinical picture.

METHODS: To report the case of an 80 year-old patient, admitted to the Department of Gastroenterology with anaemia associated with diarrhea for one week. After blood, feces and image tests a solid mass was detected in the left renal parenquima; she was referred to our service and radical nephrectomy was performed. After 8 months of follow up her outcome is satisfactory.

RESULTS: Renal carcinoid is an infrequent entity, included among neuroendocrine tumors, described 100 years ago by Lubart, derived from Kuchitsky's cells, they produce polipeptidic hormones and biogenic amines; being more frequent in the gastrointestinal tract (62-67\%) and respiratory tract(22-27\%). Generally, these tumors have a favorable outcome when they are diagnosed in time.

CONCLUSIONS: Primary renal carcinoid is an infrequent entity among endocrine tumors. We must in-dicate this case's presentation was with anemia, diarrhea and positive occult blood in feces.

Keywords: Renal disease. Renal carcinoma. Carcinoid. 


\section{INTRODUCCIÓN}

El Tumor Carcinoide (TC) es una patología extremadamente rara, que engloba un manejo multidisciplinario, por su forma de presentación. En ocasiones aparece con síntomas y signos de otro aparato, sobre todo digestivo donde es más frecuente su aparición. Estimándose que el $50-95 \%$ de ellos desarrollan metástasis hepáticas y el $80 \%$ con afección avanzada fallecen a los 5 años. Apesar de ello, estos tumores no son considerados muy agresivos con relación a otros.

El diagnóstico se basa en la característica de producir diversos péptidos hormonales (funcional) y en la frecuente presencia de receptores de somatostatina en la superficie del tumor. El uso de la gammagrafía con Octreotido nos da el diagnóstico de localización; el estudio anátomo patológico se realiza por estudio histo-químico y la identificación sobre todo de la cromogranina $A$.

Tiene dos formas de tratamiento: quirúrgico, que es la elección principal y la conducta farmacológica.

\section{PRESENTACIÓN DEL CASO}

Mujer de 80 años de edad con antecedentes de hernia hiatal, glaucoma, cataratas intervenidas, valvulopatías mitral y aórtica con hipertrofia ventricular izquierda; hipertensión arterial, dislipedemia, diabetes mellitus no insulino requiriente e hiperuricemia, que ingresó en el Servicio de Digestivo por presentar un síndrome anémico, asociado a cuadro diarreico de una semana de evolución.

Durante su evaluación, se realiza ecografía abdominal hallándose incidentalmente una masa sólida de $4.2 \mathrm{~cm}$. de diámetro, con aspecto nodular, homogénea, ligeramente hipoecoica en relación con el parénquima renal. Con éste hallazgo es remitida al Servicio de Urología donde se le indica una tomografía axial computarizada de abdomen y pelvis demostrándose masa de $4 \mathrm{~cm}$. en polo inferior del riñón izquierdo, presentando realce con la administración del medio de contraste endovenoso, sin evidencia de alteraciones vasculares, presencia de adenopatías retroperitoneales ni mesentéricas. Una evaluación completa para descartar metástasis fue realizada, incluyendo Rx. de tórax, todo lo cual fue negativo.

En el mes de febrero de 2008 fue llevada a quirófano realizándole nefrectomía radical abierta sin complicaciones. El estudio histopatológico final reportó la presencia de un tumor en el polo inferior del riñón, de $4 \mathrm{~cm}$. de diámetro, extensamente hemorrágico, bien delimitado y con afectación hasta la cápsula renal (Figuras 1 y 2). Se le practica NSE, Cromogranina y Synaptopysina resultando positivos (Figuras 3 y 4). No se observó invasión pielocalicial, a la vena renal, ni del tejido adiposo de la celda renal, concluyendo tumor carcinoide primario de riñón.
No hubo complicaciones post operatorias, desapareciendo la sintomatología gastrointestinal por lo cual había ingresado la paciente, una semana después de haber realizado la cirugía.

\section{DISCUSIÓN}

Los tumores carcinoides fueron descritos hace aproximadamente 100 años por Lubart (7). Algunos piensan que tienen un origen neuroendocrino, derivando de las células de Kulchitsky (7), donde se observan al microscopio electrónico numerosos gránulos de secreción los cuales se caracterizan por la producción de hormonas polipéptidas y aminas biógenas, así como de serotonina, histamina, dopamina, neurotensina, corticotropina, sustancia $\mathrm{P}$, calicreina y prostaglandina, aunque la sustancia mas frecuentemente hallada es la serotonina, que se transforma por intermedio de la monoaminoxidasa en 5 hidroindolacetico. La reactividad de los gránulos citoplasmaticos argirofilos (2-7) a las tinciones de plata como el grimleius y la inmunoreactividad a la enolasa, además de la cromogranina $\mathrm{A}$ hacen el diagnóstico histológico (2-5).

Estos tumores se localizan con más frecuencia en el aparato digestivo (62-67\%) y respiratorio $(22-27 \%)(2-7)$. Revisando la literatura, se han publicado hasta el año 2006 sólo 40 casos de manera primaria en riñones, apareciendo en el $23 \%$ en riñones en herradura (7).

El $25 \%$ de los tumores se presentan en el momento del diagnóstico con metástasis a pulmón, ganglios, hígado - huesos. Asumimos que el cuadro digestivo de la paciente estuvo asociado a la liberación de las aminas anteriormente descritas, por el tumor. Una vez realizada la nefrectomía desaparecieron los síntomas digestivos.

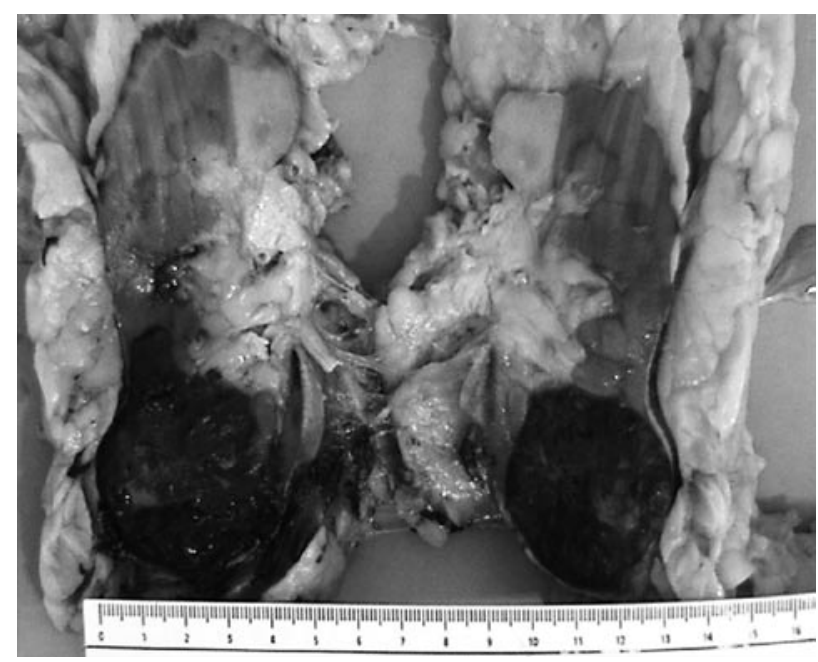

FIGURA 1. Macroscópico: pieza de nefrectomía izquierda con tumoración polo inferior. 


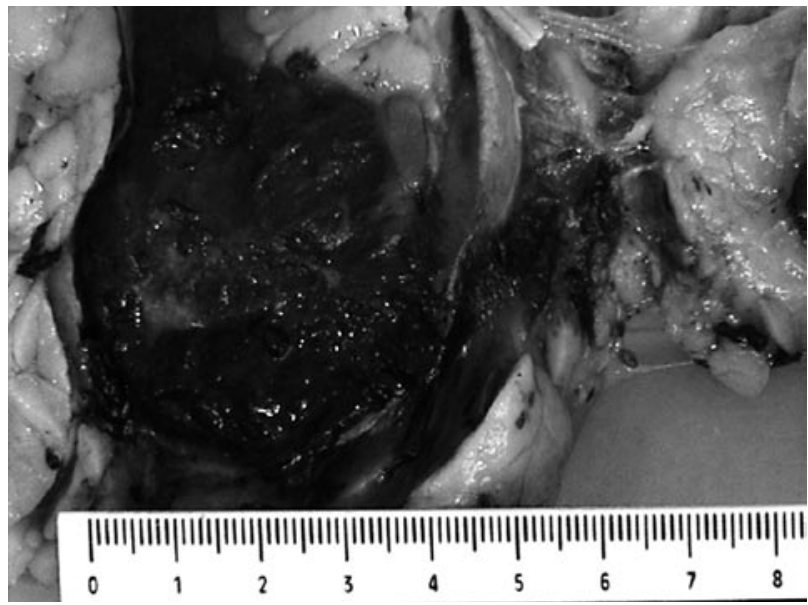

FIGURA 2. Macroscópica: Formación tumoral de $4 \mathrm{~cm}$. de diámetro, bien delimitada y hemorrágica.

En el tumor renal carcinoide localizado, el tratamiento de elección es la extirpación quirúrgica. Varios reportes, incluyendo los de Cole et al. han demostrado que la nefrectomía radical abierta y parcial han curado con éxito el tumor renal carcinoide primario. Más recientemente, Lowrance et al. describen la factibilidad de la nefrectomía parcial por laparoscopia en el manejo de una paciente de 48 años con el diagnóstico de un tumor renal izquierdo de $3 \mathrm{~cm}$. diagnosticado incidentalmente durante la evaluación de síntomas gastrointestinales.

Cuando el tumor carcinoide es menor de $1 \mathrm{~cm}$. la incidencia de metástasis es baja.

En caso de enfermedad metastásica se ha utilizado Radioterapia y Quimioterapia (platino-etoposido) con malos resultados (7). También se ha utilizado el Octreotide, demostrando una remisión parcial en el $8 \%$ de los casos (7), apreciándose una disminución en la enfermedad metastásica de un $50 \%$ a un $19 \%$ a los 5 años en Holanda (4).

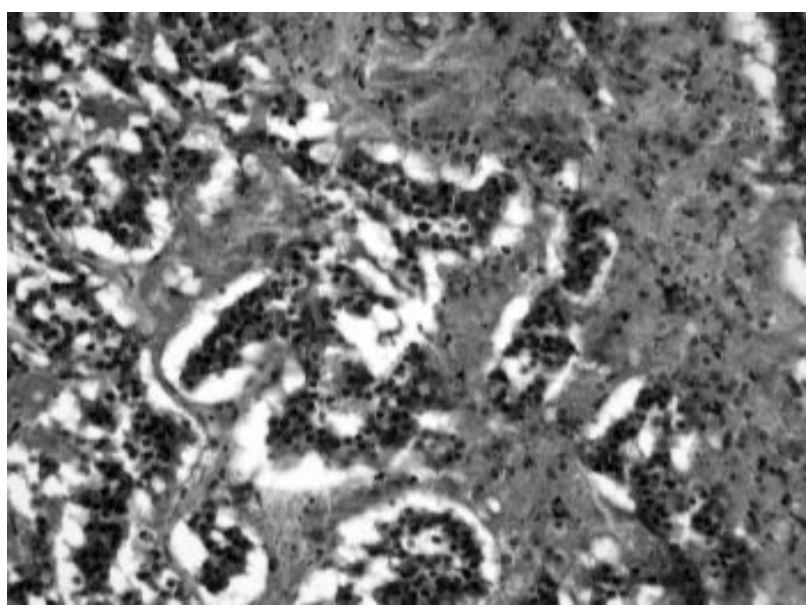

FIGURA 4. Microscópico: Cromogranina positiva.

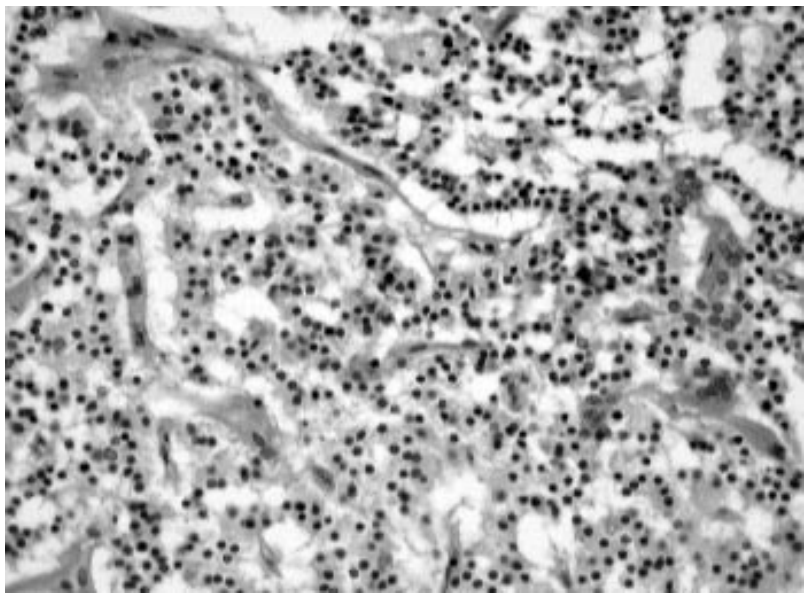

FIGURA 3. Microscópica: Formación tumoral de patrón trabecular, con núcleos regulares, sin atipia ni mitosis. (M-E).

\section{CONCLUSIÓN}

El tumor carcinoide primario de riñón es una lesión poco frecuente. Su presentación suele ser similar a la del hipernefroma, asociándose frecuentemente síntomas derivados de la liberación de aminas vasoactivas.

El tratamiento consiste en la nefrectomía radical y su evolución suele ser favorable.

\section{BIBLIOGRAFÍA y LECTURAS RECOMENDADAS ( ${ }^{*}$ lectura de interés $y^{* *}$ lectura fundamental)}

1. Mallén Mateo E, Sancho Serrano C, Gil Sanz MJ, Borque Fernando A, Jagüe Romeo D, Rioja Sanz LA: Teratoma renal: Aportación de un caso y revisión de la Literatura. Actas Urol 2005; $29: 5$.

2. Andrés C, Ossa J: Tumor carcinoide primario de riñón. Fundación Cardio-Infantil Universidad El Bosque, Bogotá. D.C. Colombia, Marzo 2006.

3. Cole EE, De Souza R, Schafell, Cookson MS. Primary renal carcinoid tumor. J Urol 2004; 171: 338.

4. Kawakiri $\mathrm{H}$ et al. Carcinoid tumor of a kidney presenting as a large abdominal mass: report of a case. Surg Today 2004; 34: 8689.

5. Elizande Apestegui I, Borda Celaya F. Tumor y síndrome carcinoide. (tomado de www.aegastro.es)

6. Mejor pronóstico para tumores carcinoides en Holanda. Babs G, Taal. Netherlands Cancer Institute/ Antoni Van Leewvenhoek Hospital, Amsterdan, Paises Bajos, Agosto 2006.

7. Hansel Donna E, Epstein JI et al. Renal carcinoid tumor: A clinicopathologic study of 21 cases. 2007; 31: 1539-44.

8. Mallén E, Sancho Serrano C, Gil Sanz MJ et al. Teratoma renal, aportación de un Caso y revisión de la literatura. Actas Urol 2005; 29. 
9. Grinberg A, Carro G. Poliquistosis renal, Hiperparatiroidismo y carcinoide de duódeno, Reunión Anátomo-Clínico, Instituto de Investigaciones Médicas Alfredo Lanari el 6/X/2000.Medicina (B. Aires)V. 62 n. Buenos Aires /Julio-Ag 2002

10. Moro Rodríguez E. Anatomía Patológica de los tumores pediátricos malignos más frecuentes. Introducción y clasificaciones (Parte 1 de 5). Monografía 8.199

11. Andreu C, Novick SC: Campbell. Tumores Renales. Campbell Urología. $8^{a}$ edición. 2005; Tomo 4. Cap 75. Pag. 2914. 